\title{
Barreiras de acesso à saúde da criança em uma comunidade ribeirinha da Amazônia Ocidental
}

\author{
Barriers to access to children's health in a Western Amazon riverside community
}

Barreras al acceso a la salud infantil en una comunidad ribereña en la Amazonía Occidental

Francidani Rocha Gemaque ${ }^{1}$, Wanessa Barbosa Oliveira Lima ${ }^{1}$, Marcela Milrea Araújo Barros ${ }^{1 *}$.

\section{RESUMO}

Objetivo: Analisar as barreiras do familiar/cuidador para o acesso aos serviços de saúde à criança em uma comunidade ribeirinha da Amazônia Ocidental. Métodos: Realizou-se uma pesquisa de abordagem qualitativa, descritiva a partir da técnica entrevista com roteiro semiestruturado. A análise foi realizada a partir da análise de conteúdo. Resultados: Participaram do estudo 20 familiares/cuidadores dos quais 15 mães, 2 pais e 3 avós de crianças do nascimento aos 12 anos de idade residentes na comunidade. Como barreiras de acesso à saúde da criança, as evidências estão relacionadas à localização geográfica e a ausência de unidade de saúde na comunidade, fazendo com que os moradores tenham que se deslocar até a unidade mais próxima, via fluvial em pequenas embarcações. Existem deficiências relacionadas à vacinação, consultas de puericultura e saúde bucal. Conclusão: Há a existência de vulnerabilidades programáticas e sociais na comunidade infantil, é imperativo a articulação de estratégias que promovam o acesso à saúde para crianças ribeirinhas.

Palavras-chave: Saúde, Família, Comunidade, Criança, Enfermeiro.

\begin{abstract}
Objective: To analyze the family/caregiver's barriers to accessing child health services in a riverside community in the Western Amazon. Methods: A qualitative, descriptive research was carried out using the interview technique with a semi-structured script. The analysis was carried out from the content analysis. Results: Twenty family members/caregivers participated in the study, including 15 mothers, 2 fathers and 3 grandparents of children from birth to 12 years of age living in the community. The barriers to accessing children's health, the evidence is related to geographic location and the absence of a health unit in the community, causing residents to travel to the nearest unit, by river in small boats. There are deficiencies related to vaccination, childcare consultations and oral health. Conclusion: There is the existence of programmatic and social vulnerabilities in the children's community, it is imperative to articulate strategies that promote access to health for riverside children.
\end{abstract}

Keywords: Health, Family, Community, Child, Nurse.

\section{RESUMEN}

Objetivo: Analizar las barreras de la familia/cuidador para acceder a los servicios de salud infantil en una comunidad ribereña de la Amazonía Occidental. Métodos: Se realizó una investigación descriptiva cualitativa mediante la técnica de entrevista con guión semiestructurado. El análisis se realizó a partir del análisis de contenido. Resultados: Veinte familiares/cuidadores participaron en el estudio, incluidas 15 madres, 2 padres y 3 abuelos de niños desde el nacimiento hasta los 12 años que viven en la comunidad. Las barreras para acceder a la salud de los niños, la evidencia está relacionada con la ubicación geográfica y la ausencia de una unidad de salud en la comunidad, provocando que los residentes se desplacen a la unidad más cercana, por río, en pequeñas embarcaciones. Existen deficiencias relacionadas con la vacunación, las consultas de puericultura y la salud bucal. Conclusión: Existe la existencia de vulnerabilidades programáticas y sociales en la comunidad infantil, es imperativo articular estrategias que promuevan el acceso a la salud de los niños ribereños.

Palabras clave: Salud, Familia, Comunidad, Niño, Enfermera.

1 União das Escolas Superiores de Rondônia (UNIRON), Porto Velho - RO.

*E-mail: marcela.barros@uniron.edu.br

SUBMETIDO EM: 6/2021

ACEITO EM: 6/2021

PUBLICADO EM: 7/2021 


\section{INTRODUÇÃO}

O conceito de acesso à saúde está ligado aos princípios de equidade, integralidade e universalidade do Sistema Único de Saúde (SUS). Esse acesso é um dos maiores desafios do sistema público de saúde, tanto no Brasil quanto em outros países. O mesmo está relacionado a garantia de atendimento em tempo oportuno, com informação, no horário mais confortável ao usuário, com equipe de referência de forma contínua e resolutiva (CIRINO FMSB, et al., 2020).

Dentre o público assistido pelos serviços de saúde, torna-se importante, compreender como se dá o acesso para o público infantil. Muitos esforços têm sido empreendidos para melhorar o acesso à criança nos serviços de saúde e assim, reduzir a morbidade e a mortalidade, pois, de acordo com a Organização Mundial de Saúde (2008), a saúde infantil é prioritária para o desenvolvimento humano. Logo, este público deve ter acesso garantido, com ações de prevenção de riscos, agravos e doenças, bem como ações de promoção, proteção, cura e reabilitação da saúde (FURTADO MCC, et al., 2018).

De acordo com a Política Nacional de Atenção Integral à Saúde da Criança do Ministério da Saúde (PNAISC), o acompanhamento da criança configura-se um dos pilares mais importantes da saúde infantil. Há inúmeras evidências científicas que norteiam as diretrizes do cuidado e atenção integral para a prevenção de doenças na infância (BRASIL, 2018).

Estudos apontam a existência de barreiras de acesso aos serviços de saúde e estas, estão relacionadas principalmente aos aspectos organizacionais e as fragilidades na articulação entre os diferentes níveis e locais de prestação de serviços. Estas barreiras ficam ainda mais evidentes quando se trata da comunidade ribeirinha, pois necessitam se deslocar por via fluvial, em pequenas embarcações (ASCENSO AMR e AGUIAR RS, 2020). O ribeirinho é o indivíduo que vive às margens dos rios e lagos, sobrevive da caça, do roçado, agricultura familiar e do extrativismo de forma geral (FRANCO EC, et al., 2015). As populações ribeirinhas descendem de povos indígenas miscigenados com migrantes, principalmente o povo nordestino (GUIMARÃES AF, et al., 2020).

$A$ atenção à saúde as comunidades ribeirinhas é realizada através da Política Nacional de Atenção Básica (PNAB), revisada por meio das Portarias do Ministério da Saúde (MS) n.․ 2.436, de 21 de setembro de 2017, bem como as especificidades das Equipes de Saúde das Famílias Ribeirinhas (ESFR), e subsídio das Unidades Básicas de Saúde Fluviais (UBSF), especialmente na região amazônica. De acordo com a PNAB, a Estratégia Saúde da Família (ESF) é uma estratégia prioritária (BRASIL, 2017).

Torna-se relevante entender como se desenvolve o acesso à saúde a esse grupo populacional específico. Para tanto, a questão norteadora deste estudo foi: Quais as barreiras enfrentadas pelos familiares de crianças ribeirinhas para o acesso aos serviços de saúde? Nesta perspectiva, o estudo objetiva analisar as barreiras do familiar/cuidador para o acesso aos serviços de saúde à criança em uma comunidade ribeirinha da Amazônia Ocidental.

\section{MÉTODOS}

Trata-se de uma pesquisa de abordagem qualitativa, descritiva, a partir do uso da técnica entrevista com roteiro semiestruturado. A pesquisa foi realizada com o familiar/cuidador de crianças residentes em uma comunidade ribeirinha localizada na Região Norte do Brasil, Amazônia Ocidental.

Foram considerados como critérios para inclusão o participante (familiar/cuidador, ou seja, mãe, pai, avô e avó) morador da comunidade ribeirinha que se disponibilizasse a participar voluntariamente do estudo, possuir criança no domicílio desde o nascimento aos doze anos de idade, independentemente do número de crianças no seu núcleo familiar. Foram excluídos àqueles com idade inferior a 18 anos.

Após aproximação com o cenário de pesquisa, foi realizado visita domiciliar utilizando-se da amostragem do tipo bola de neve, não probabilística no qual se usam cadeias de referência. Assim, a partir desse tipo de amostragem não é possível determinar a probabilidade de seleção de cada participante na pesquisa, mas torna-se útil para estudar determinados grupos difíceis de serem acessados (VINUTO JA, 2014). A 
entrevista com roteiro semiestruturado de seis questões foi realizada no próprio domicílio do participante, em ambiente reservado com tempo aproximado de quinze minutos.

A análise de dados foi realizada com base na análise de conteúdo do tipo categorial ou temática (MINAYO MCS, 2010). Para assegurar o anonimato dos participantes, os nomes ficaram em sigilo, por meio do uso de códigos definidos pela letra $A$ e números de 1 a 20. A pesquisa iniciou-se após a aprovação do Comitê de Ética e Pesquisa, com parecer consubstanciado n.․․ 4.316 .896 e número do CAAE 37076920.0 .0000 .8028 em dois de outubro de 2020. Foram respeitadas as exigências preconizadas pela Resolução $n^{\circ}$ 466/12 de 12 de dezembro de 2012 do Conselho Nacional de Saúde/Ministério da Saúde que dispõe sobre pesquisas envolvendo seres humanos.

\section{RESULTADOS E DISCUSSÃO}

Participaram do estudo 20 cuidadores familiares de crianças moradoras na comunidade dois quais, 15 foram mães, 3 avós e 2 pais, num total de 18 mulheres e 2 homens. A faixa etária mais prevalente das crianças é de um a cinco anos, com aproximadamente duas crianças por família. Quanto ao local de nascimento das crianças da comunidade, todas nasceram na capital do Estado (Tabela 1).

Tabela 1 - Caracterização dos familiares/cuidadores moradores da comunidade ribeirinha.

\begin{tabular}{lll}
\hline Variável & N & $\%$ \\
\hline Sexo do familiar/cuidador & 18 & \\
\hline Feminino & 02 & 85 \\
Masculino & & 15 \\
\hline Grau parentesco do familiar/cuidador & & \\
com a criança & 15 & 75 \\
\hline Mãe & 03 & 15 \\
Avó & 02 & 10 \\
Pai & & \\
\hline Local de nascimento da criança que o & & 100 \\
familiar/cuidador é responsável & 32 & 00 \\
\hline Capital do Estado & 00 & \\
Outra localidade & & 6,3 \\
\hline Faixa Etária da criança que o familiar/ & & 59,4 \\
cuidador é responsável & 02 & 21,8 \\
\hline Menor de um ano & 19 & 12,5 \\
1-5 & 07 & \\
6-9 & 04 &
\end{tabular}

Fonte: Gemaque FR, et al., 2021.

Como resultado da análise dos dados emergiram quatro categorias temáticas: Barreiras de acesso aos serviços de saúde na Comunidade; Estratégias apontadas pelos cuidadores em situações de urgência ou emergência; Necessidades de saúde à criança ribeirinha e; O enfermeiro como ator estratégico para o acesso aos serviços de saúde à criança.

\section{Barreiras de acesso aos serviços de saúde na comunidade}

A constituição de uma rede regionalizada, capaz de atender as populações nas regiões mais longínquas do país, tem sido um dos principais desafios ao longo dos 30 anos do Sistema Único de Saúde (SUS). O atendimento em regiões de difícil acesso, como é o caso de várias comunidades da Amazônia ocidental, entre elas a comunidade investigada, exigem arranjos institucionais peculiares, visto que a criação de Unidades Básicas de Saúde (UBS) depende de pactuações federativas entre o governo estadual e municipais que as constituem, e ainda de acordos entre os Secretários Municipais (OLIVEIRA RAD, et al., 2019). 
Os relatos dos participantes moradores da comunidade ribeirinha referem a ausência de uma UBS, constituindo-se, portanto, a principal barreira de acesso como mínimo essencial na perspectiva do direito à saúde da criança:

"O posto de saúde encontra-se fechado. Precisa se deslocar para outra comunidade. Depende de ter gasolina e apoio de parentes ou vizinhos para se deslocar" (A1).

"Antes tinha agente de saúde e agendava, uma médica vinha de mês em mês aqui. Tem que ir para outra comunidade" (A16).

"Tem o postinho que nós mesmos construímos, mas não tem ninguém pra trabalhar lá. Está fechado" (A16).

Os resultados apontam para uma realidade sem acesso a UBS, faltam profissionais para compor uma equipe de saúde. Tais relatos demonstram que a realidade está em desacordo com a PNAB, a qual prevê que a prevenção de agravos, a investigação, tratamento, recuperação, promoção e proteção da saúde, são compreendidas pela APS que é o primeiro nível de atenção à saúde, representada por um conjunto de ações no âmbito individual e coletivo (BRASIL, 2011).

As barreiras de acesso também são enfrentadas quando a família e sua criança necessitam se deslocar para outra comunidade ou distrito mais próximo para uma consulta.

"Conseguir consulta. Não tem pediatra na comunidade, e nem em outra comunidade. Não conseguimos pegar remédio para as crianças. Às vezes acontece de irmos lá à outra comunidade e voltarmos sem conseguir a consulta" (A2).

"Primeiro que temos que sair daqui para ir para outra comunidade. Para conseguirmos atendimento dependemos dos outros pois não temos rabeta" (A20).

Os entrevistados citam a falta de pediatra, medicações e dificuldades para o transporte até outra comunidade mais próxima. A rabeta descrita pelo participante é um tipo de barco pequeno com motor de propulsão, acionado manualmente com o auxílio de um equipamento de direção. As falas, portanto, representam as várias dificuldades enfrentadas pelos ribeirinhos que estão em desarmonia com o que determinado pela legislação vigente (BRASIL, 2018).

Ao fazer referência acerca da maior dificuldade para o atendimento de saúde às suas crianças, os entrevistados referem à distância para o deslocamento até o distrito mais próximo que possua uma unidade de saúde, a falta de transporte e o preço do combustível, pois, precisam custear esse transporte:

"Até chegar em outra comunidade, já morreu" (A3).

"A maior dificuldade é não conseguir consulta, conseguimos o combustível que é muito caro e damos viagem perdida. Só gastamos combustível" (A17).

O acesso não equivale à simples utilização do serviço de saúde, mas também à oportunidade de dispor dos serviços em circunstâncias que permitam o uso apropriado dos mesmos, no tempo adequado, para o alcance dos melhores resultados de saúde (SILVA CR, et al., 2017). A precariedade de acesso dos ribeirinhos aos serviços de saúde pública revela a necessidade de medidas amplas de promoção de saúde, associada à oferta de serviços adequada à realidade das comunidades ribeirinhas distantes dos espaços urbanos (JÚNIOR AMF, et al., 2020).

Embora as políticas ministeriais possuam uma atenção focalizada para as populações vulneráveis, essa universalidade do SUS não chega à comunidade na sua totalidade. Os problemas de cunho econômico atrelados ao distanciamento e a dificuldade de transporte são potencializados pelos familiares/cuidadores:

"Dependemos de outras pessoas pra ir para outra comunidade, gastamos combustível, tem até uma lancha no porto, mas não tem motorista" (A15). 
"Nós temos barco próprio, mas o combustível aqui tem um custo muito alto, não é sempre que temos dinheiro" (A16).

As limitações de acesso aos serviços de saúde compõem um contexto multifatorial, complexo e subjetivo, que podem implicar em diferentes aspectos no modo de vida dessas populações (GUIMARÃES $A F$, et al., 2020). A precariedade de transportes é um fato em todas as regiões ribeirinhas, pois, há desinteresse das lanchas e da aviação comercial, tendo vista o alto custo, longa distância para pouca procura do serviço, limitando a quantidade de viagens fluviais. Quanto à possibilidade de construções de estradas, são inviáveis devido às condições ambientais, sendo a construção de estradas de alto custo (FERREIRA D, et al., 2020).

Nesse sentido, percebe-se a vulnerabilidade social vivenciada pelas comunidades ribeirinhas da Amazônia quando o assunto é acesso à saúde, no qual se observam cenários de pobreza, baixo nível escolar, moradias precárias e participação em programas sociais. Além disso, evidencia-se também, a existência da vulnerabilidade programática, pelas fragilidades na gestão dos programas de saúde, dos serviços ofertados e demandas necessárias. O conceito de vulnerabilidade propõe um novo olhar para as necessidades de saúde, incorporando, para além dos saberem epidemiológicos e biomédicos, os saberes oriundos dos campos das ciências sociais e humanas (CALAZANS GJ, et al., 2018). Face ao abordado, o sentimento de esquecimento pelos órgãos gestores de saúde e não pertencimento a sociedade é descrito por um dos moradores da comunidade:

"A ideia de esquecimento e não pertencimento à sociedade fica muito explícito na frase 'aqui não tem nada"' (A7).

O governo brasileiro, com o intuito de minimizar as desigualdades de acesso de algumas populações, entre elas, a população ribeirinha, apresentou em 2013, como estratégia o Programa Mais Médico, o qual levou médicos para as regiões vulneráveis do Brasil, incluindo a população ribeirinha. Porém, mesmo com esta cobertura de médicos oferecida, não foi o suficiente para assistir integralmente a população alvo (GUIMARÃES AF, et al., 2020).

\section{Estratégias apontadas pelos cuidadores em situações de urgência ou emergência}

Quanto às dificuldades encontradas na comunidade na ocorrência de alguma urgência ou emergência com suas crianças, de acordo com os entrevistados, no distrito mais próximo não há atendimento todos os dias, enfrentando assim a dificuldade de deslocamento até a área urbana nas situações de urgência, o que pode durar até seis horas, caso seja realizado por motor de propulsão:

"Quando é muito grave, a gente leva a criança direto para a capital com meios próprios. Então são muitas dificuldades, é o barco, o combustível, o carro para a estrada" (A6).

"Não tem transporte, aqui não tem nada, se você não conseguir o barco, a criança vai morrer aqui" (A12).

"Não tem ambulancha, depende de outras pessoas pra ir pra São Carlos gasta gasolina, tem até uma lancha no porto, mas não tem motorista" (A15).

Os relatos vão de encontro com o que é disposto pela Portaria Ministerial o 824 no qual estabelece as diretrizes da regulação médica das urgências que determina ser indispensável a implementação de uma assistência oportuna com transporte apropriados de acordo com os princípios e diretrizes do SUS. (GARNELO L, et al., 2018).

Para esse tipo de situação e população (indígenas e ribeirinhos) deveria existir o Serviço de Atendimento Móvel de Urgência (SAMU), por via fluvial através de ambulanchas, normatizado em 2004, o qual é operacionalizado por meio de embarcações de atendimento básico, com condutor e técnico de enfermagem 
e o avançado com médico, enfermeiro e condutor-socorrista (FERREIRA D, et al., 2020). Porém, na comunidade investigada não há ambulancha, indicando assim, que a concepção de um sistema universal, igualitário e integral ainda não se concretizou, o que sinaliza que deliberações políticas são essenciais para o fortalecimento do SUS (SILVA AP, et al., 2020).

\section{Necessidades de saúde à criança ribeirinha}

O crescimento e desenvolvimento infantil são reconhecidos como um importante indicador na área da saúde pública para monitorar o estado de saúde e nutrição de populações, em razão de sua estreita dependência de fatores ambientais (SILVA AP, et al., 2020). Ainda que o Programa Nacional de Imunização (PNI) seja explícito ao recomendar que a vacinação ocorra dentro da idade correta, pois a cobertura vacinal é considerada um importante indicador de saúde, esta, não é a realidade vivida pelos moradores da comunidade. Vacinar a criança não é algo que se tenha acesso com facilidade, conforme afirmam familiares cuidadores sobre as principais necessidades de saúde:

"Vacinar é uma necessidade para nossas crianças" (A5).

"Foi muito difícil conseguir vacinar as crianças. Esse meu filho menor, foi difícil porque nem toda às vezes tinha vacina lá na outra comunidade. Quando tinha vacina não tinha o vacinador" (A19).

A vacinação é um direito que deve ser respeitado, protegido e assegurado pelo Estatuto da Criança e do Adolescente e pelos princípios de justiça social (BRASIL, 2012). A puericultura é outra necessidade contida nos depoimentos dos entrevistados:

"Difícil para quem tem criança, tem que esperar a vaga, tem muito adulto para se consultar. É difícil fazer exames de rotina nas crianças. Nos meus filhos só fiz quando eles nasceram, exames de fezes, urina e hemograma só fizemos quando os acadêmicos vinham, era uma ajuda para nós" (A8).

De acordo com o Caderno de Atenção Básica para a saúde da criança, pela permanência das desigualdades loco regionais, ainda não foi possível atingir a meta de que toda criança brasileira tenha assegurado o direito à vida e à saúde. Nesse contexto, a puericultura é estratégica, pois possibilita o acompanhamento e avaliação de riscos de morbidade e mortalidade que podem afetar sua saúde atual ou a qualidade de vida quando adulto (BRASIL, 2012).

Uma sugestão para avaliar a puericultura e seu desenvolvimento é o projeto Barco da Saúde, que realiza esse tipo de trabalho em algumas regiões ribeirinhas, tendo em vista que esse acompanhamento às crianças é essencial para identificação de risco nutricional e neuropsicomotor, o que determina a necessidade de maior atenção à saúde da criança (JÚNIOR AMF, et al., 2021).

Outra necessidade de saúde relatada pelos entrevistados é a falta de atendimento e acompanhamento da saúde bucal das crianças:

"Busca por dentista. As crianças estão precisando, mas ainda não consegui, pois quando fui não tinha dentista" (A1).

O momento da infância é estratégico para a saúde bucal, pois, é a faixa etária ideal para desenvolver hábitos saudáveis e para participação em programas educativo/preventivos de saúde bucal. Podem ser desenvolvidos diversos trabalhos na APS, com enfoque familiar importante para o aprendizado que ocorre por meio da observação do comportamento dos pais (NETTO MFV e CHICON JB, 2020).

A falta de acesso a serviços de saúde bucal é evidente na população ribeirinha, onde muitos têm uma perda significativa da dentição muito cedo, o que poderia ser evitado se houvesse iniciativa política voltada para o cuidado da dentição na infância, principalmente (GARNELO L, et al., 2018). Esse dado se confirma no estudo de Amaral RC, et al. (2017), o qual avaliou os índices de saúde bucal em oito comunidades ribeirinhas, e verificou as mesmas dificuldades e a perda de dentição precoce. 
Netto MFV e Chicon JB, (2020) concluem que é necessário adotar medidas urgentes de atenção à saúde bucal das crianças ribeirinhas, sendo imprescindível a promoção de ações que ultrapassem as barreiras e dificuldades de locomoção e governamentais, valorizando a saúde bucal como um bem para a qualidade de vida de todos os cidadãos.

\section{O enfermeiro como ator estratégico para o acesso aos serviços de saúde à criança}

Importante registrar que a política de saúde da criança é resultado de um longo processo de aperfeiçoamento e evolução das políticas públicas nacionais. Este processo está diretamente ligado com a qualificação da assistência ofertada em todos os níveis de atenção à saúde, com a atuação de diferentes atores, entre eles, o profissional enfermeiro (BRANQUINHO ID e LANZA FM, 2018).

A atuação do enfermeiro na APS como integrante de uma equipe, vem se destacando por se mostrar estratégico e fundamental para integralidade do cuidado, educação em saúde, diminuindo os fatores de risco, prevenindo doenças e promovendo a saúde e a qualidade de vida da população (FERREIRA SRS, et al., 2018).

Ao serem abordados sobre quais as propostas de melhorias no acesso aos serviços de saúde oferecidos à sua comunidade, grande parte dos familiares cuidadores consideram que ativar o posto de saúde existente na comunidade é a melhoria mais urgente e que iria ao encontro dos anseios e necessidades dos moradores, no qual, o enfermeiro, como membro da equipe de saúde, poderia ter um olhar específico para o cuidado à saúde das crianças, conforme responderam:

\section{"Precisamos de um enfermeiro e que o posto de saúde funcione" (A11). \\ "Com um enfermeiro, ajudaria muito. Precisamos dele para poder atender as crianças" (A1).}

"Ajudaria com certeza pelo menos para vacinar as crianças" (A3).

Ao tomar como base outros estudos realizados com populações ribeirinhas da Amazônia e em países como Austrália, o qual se caracteriza também pelas diferenças sociopolíticas, geográficas e culturais, as populações rurais, e também sofrem consequências negativas impostas pelas mesmas questões que podem limitar o alcance aos serviços de saúde por essas populações (GUIMARÃES, AF et al., 2020).

De acordo com Júnior AMF, et al., (2020), os ribeirinhos não se atentam para buscar prevenção, diagnóstico ou tratamento de uma determinada doença, isso é justificado pela falta de informação ou dificuldade de acesso à saúde, ou seja, se essas ferramentas fossem facilitadas, o panorama seria diferente. Nesse sentido, a atuação do enfermeiro, como líder da ESF, vem se solidificando como ferramenta de mudança da prática assistencial para concretização do princípio do SUS, legitimando a proposta de um novo modelo de atenção à saúde que não se concentre apenas na clínica e no tratamento, mas também na atenção integral e nas intervenções frente aos agravos, prevenção de doenças e promoção da saúde com ações de qualidade (FERREIRA SRS, et al., 2018).

\section{CONCLUSÃO}

As principais barreiras de acesso à saúde das crianças ribeirinhas apontadas pelos familiares/cuidadores, referem-se às características geográficas da região e ausência de unidade básica de saúde, exigindo o seu deslocamento para atendimento. Como necessidades de saúde, registram-se principalmente ações de imunização, puericultura e saúde bucal. É determinante se repensar os formatos de articulação e implementação de estratégias que promovam o acesso aos serviços de saúde às crianças ribeirinhas. Acredita-se que estudos direcionados a esse grupo com vulnerabilidades sociais e programáticas se fazem oportunos para o crescimento da produção e difusão do conhecimento, com ênfase no papel do enfermeiro, como um ator estratégico junto à equipe multiprofissional, nas ações de prevenção, promoção e um cuidado centrado na integralidade junto ao binômio família e sua criança ribeirinha. 


\section{REFERÊNCIAS}

1. AMARAL RC, et al. A relação entre a saúde bucal e a cárie dentária em oito comunidades ribeirinhas - Pará, Brasil. Rev. Bras. Odontol., 2017; 74(1): 18-22.

2. ASCENSO AMR, AGUIAR RS. Acesso da criança na atenção primária à saúde: uma revisão integrativa. Saúde coletiva, 2020; 10 (59): 4465-4473.

3. BRANQUINHO ID, LANZA FM. Saúde da Criança na Atenção Primária: Evolução das Políticas Brasileiras e a Atuação do Enfermeiro. Revista de Enfermagem do Centro-Oeste Mineiro, 2018; 8: e2753.

4. BRASIL. Ministério da Saúde. Secretaria de Atenção à Saúde. Departamento de Atenção Básica. Saúde da criança: crescimento e desenvolvimento- Cadernos de Atenção Básica, no 33- Brasília: 2012. Disponível em: https://bvsms.saude.gov.br/bvs/publicacoes/saude_crianca_crescimento_desenvolvimento.pdf. Acessado em: 10 de maio de 2021.

5. BRASIL. Ministério da Saúde. Secretaria de Atenção à Saúde. Área Técnica de Saúde da Criança e Aleitamento Materno. Gestões e gestores de políticas públicas de atenção à saúde da criança: 70 anos de história. Brasília: Ministério da Saúde, 2011. (Série I. História da saúde). Disponível em: http://bvsms.saude.gov.br/bvs/publicacoes/70_anos_historia_saude_crianca.pdf. Acessado em: 10 de maio de 2021.

6. BRASIL. Ministério da Saúde. POLÍTICA NACIONAL DE ATENÇÃO INTEGRAL À SAÚDE DA CRIANÇA (PNAISC). Orientações para implementação. Secretaria de Atenção à Saúde. Departamento de Ações Programáticas Estratégicas. - Brasília, 2018. 180 p.: il. ISBN 978-85-334-2596-5. Acessado em: 15 de junho de 2021.

7. BRASIL. Ministério da Saúde. Portaria no 2.436, de 21 de setembro de 2017. Aprova a Política Nacional de Atenção Básica, estabelecendo a revisão de diretrizes para a organização da Atenção Básica, no âmbito do Sistema Único de Saúde (SUS). Brasília, DF: Ministério da Saúde, 2017. Disponível em: http://www.brasilsus.com.br/index.php/legislacoes/gabinete -do- -ministro/16247portaria-n-2-436-de-21-de-setembrode-2017. Acessado em: 15 de junho de 2021.

8. CALAZANS GJ, et al. Vulnerabilidade programática e cuidado público: Panorama das políticas de prevenção do HIV e da Aids voltadas para gays e outros HSH no Brasil. Sexualidad, Salud y Sociedad, 2018; 29: 263-293.

9. CIRINO FMSB, et al. O Acesso Avançado como estratégia de organização da agenda e de ampliação do acesso em uma Unidade Básica de Saúde de Estratégia Saúde da Família, município de Diadema, São Paulo. Rev Bras Med Fam. Comunidade, 2020; 15(42):21111.

10. FERREIRA SRS, et al. The complexity of the work of nurses in Primary Health Care. Revista Brasileira de Enfermagem, 2018; 71 (1): 704-709.

11. FERREIRA D, et al. Desafios na manutenção do serviço de atendimento móvel de urgência fluvial no alto Solimões. Revista Humanidades e Inovação, 2020; 7(16): 495-505.

12. FRANCO EC, et al. Promoção da saúde da população ribeirinha da região amazônica: relato de experiência. Revista CEFAC, 2015; 17(5):1521-1530.

13. FURTADO MCC, et al. Ações e articulações do enfermeiro no cuidado da criança na atenção básica. Texto \& Contexto Enferm, 2018; 27(1): e0930016.

14. GARNELO L, et al. Acesso e cobertura da Atenção Primária à Saúde para populações rurais e urbanas na região norte do Brasil. Saúde debate, 2018; 42(1): 81-99.

15. GUIMARAES AF, et al. Acesso a serviços de saúde por ribeirinhos de um município no interior do estado do Amazonas, Brasil. Rev Pan-Amaz Saude, 2020; 11: e202000178.

16. JÚNIOR AMF. O acesso aos serviços de saúde da população ribeirinha: um olhar sobre as dificuldades enfrentadas. Revista Eletrônica Acervo Científico, 2020; 13: e4680.

17. MINAYO MCS. Pesquisa social: teoria, método e criatividade. 29. ed. Petrópolis, RJ: Vozes, 2010.

18. NETTO MFV, CHICON JB. Expedição Amazônia - promoção de saúde bucal com a população ribeirinha. Revista Científica FAESA, 2020; 16(1): 25 - 31.

19. OLIVEIRA RAD, et al. Barreiras de acesso aos serviços em cinco Regiões de Saúde do Brasil: percepção de gestores e profissionais do Sistema Único de Saúde. Cadernos de Saúde Pública, 2019; 35 (11): e00120718

20. NOVO BN, et al. Legislação do SUS. Revista Jus Navigandi, 2019; 24 (5814): 123-127.

21. ORGANIZAÇÃO MUNDIAL DE SAÚDE (OMS). Relatório Mundial de Saúde 2008. Cuidados de Saúde Primários Agora Mais Que Nunca. 2008. Disponível em: https://www.who.int/eportuguese/publications/whr08_pr.pdf?ua=1. Acessado em: 15 de junho de 2021.

22. SILVA AP, et al. Avaliação em saúde: dimensão processual e estrutural da saúde da criança na atenção primária. Saúde em Debate, 2020; 44 (124):115-129.

23. SILVA CR, et al. Dificuldade de acesso a serviços de média complexidade em municípios de pequeno porte: um estudo de caso. Ciência \& Saúde Coletiva, 2017; 22 (4): 1109-1120.

24. SOUZA RR, et al. A rede de atenção integral à saúde da criança no Distrito Federal, Brasil. Ciênc. Saúde Coletiva, 2019; 24 (6): 2075-2084.

25. VINUTO JA. Amostragem em bola de neve na pesquisa qualitativa: um debate em aberto. Temáticas, 2014; 22(44): 203-220. 